\title{
Sacubitril/valsartan for heart failure with reduced ejection fraction: A first real-life observational study in Poland
}

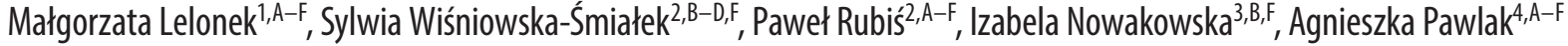 \\ 1 Department of Noninvasive Cardiology, Medical University of Lodz, Poland \\ ${ }^{2}$ Department of Cardiac and Vascular Diseases, Jagiellonian University Medical College, Kraków, Poland \\ ${ }^{3}$ Department of Invasive Cardiology, Central Clinical Hospital of the Ministry of the Interior and Administration, Warszawa, Poland \\ ${ }^{4}$ Department of Invasive Cardiology, Center of Postgraduate Medical Education, Warszawa, Poland \\ A - research concept and design; $\mathrm{B}$ - collection and/or assembly of data; $\mathrm{C}$ - data analysis and interpretation; \\ $\mathrm{D}$ - writing the article; $\mathrm{E}$ - critical revision of the article; $\mathrm{F}$ - final approval of the article
}

Address for correspondence

Małgorzata Lelonek

E-mail:malgorzata.lelonek@umed.lodz.pl

\section{Funding sources}

None declared

\section{Conflict of interest}

All authors report consultancy and lectures fees from Novartis and also participation in trials with sacubitril/valsartan.

Received on May 25, 2020

Reviewed on September 23, 2020

Accepted on 0ctober 8, 2020

\section{Cite as}

Lelonek M, Wiśniowska-Śmiałek S, Rubiś P, Nowakowska I, Pawlak A. Sacubitril/valsartan for heart failure with reduced ejection fraction: A first real-life observational study in Poland. Adv Clin Exp Med. 2021;30(1):67-75. doi:10.17219/acem/128230

DOI

10.17219/acem/128230

Copyright

Copyright by Author(s)

This is an article distributed under the terms of the

Creative Commons Attribution 3.0 Unported (CC BY 3.0)

(https://creativecommons.org/licenses/by/3.0/)

\begin{abstract}
Background. Despite the progress in the treatment of heart failure with reduced ejection fraction (HFrEF), the prognosis remains unfavorable.

Objectives. To evaluate the effectiveness, tolerance and safety after one-year follow-up of Polish patients with stable chronic HFrEF treated with sacubitril/valsartan.

Material and methods. This was an observational multicenter study conducted in 3 centers (Kraków, Łódź and Warszawa) specializing in heart failure (HF). We enrolled $89 \mathrm{HFrEF}$ patients (aged $59.3 \pm 13.5$ years, 82\% males) in NYHA class III-IV (ambulatory). Clinical, laboratory and echocardiographic parameters were evaluated at baseline and after a one-year follow-up. The composite endpoint was defined as death or urgent HF hospitalization.
\end{abstract}

Results. After 1 year, $80 \%$ of patients used $50 \%$ or more of the target dose of sacubitril/valsartan. After a year of treatment, there were significant improvements of $\mathrm{HF}$ symptoms, $\mathrm{N}$-terminal prohormone B-type natriuretic peptide (NT proBNP), ejection fraction (EF), and distance in six-minute walk test (6MWP) (all $p<0.001$ ). Patients treated with the highest dose of sacubitril/valsartan exhibited the greatest benefits. The safety profile was favorable and consistent with that previously reported; however, therapy discontinuation due to side effects occurred in 11\% of patients. The independent predictors for composite endpoint ( $n=24,26.9 \%)$ were history of HF hospitalization, tricuspid annular plane systolic excursion (TAPSE) and angiotensin-converting-enzyme inhibitor (ACEI)-naive patients.

Conclusions. Treatment of chronic HFrEF patients with sacubitril/valsartan is safe and is associated with significant clinical and objective improvement. The non-survivors had more advanced HF, so the initiation and uptitration of sacubitril/valsartan should be done early.

Key words: sacubitril/valsartan, heart failure with reduced ejection fraction, ARNI 


\section{Introduction}

The prevalence of heart failure (HF) rises exponentially and affects approx. $1-2 \%$ of the adult population; however, it can be as high as $10 \%$ in elderly patients. ${ }^{1-3}$ Patients with HF with reduced ejection fraction (HFrEF) constitute approx. $40-50 \%$ of all chronic HF patients and are broadly characterized by younger age, more prevalent coronary artery disease (CAD) and worse survival in comparison to HF with relatively preserved ejection fraction (EF). ${ }^{4}$ On the other hand, the population of HFrEF patients is much better studied, and effective and proven therapies have been successfully introduced over the last couple of decades, which have favorably improved the outcomes. ${ }^{4}$

Among effective therapies, a novel class of agents acting simultaneously on the renin-angiotensin-aldosterone system (RAAS) and the neutral endopeptidase system - angiotensin receptor neprilysin inhibitors (ARNI) - has shown promise for numerous HFrEF patients. ${ }^{5}$ In the landmark PARADIGM-HF study, ARNI was clearly superior to enalapril in improving the symptoms and prognosis for HF patients caused by ischemic or non-ischemic HFrEF. ${ }^{6}$ So far, numerous papers that clearly confirmed the benefits of sacubitril/valsartan (ARNI) in the various subgroups of HFrEF patients from different geographical settings have been published. Unfortunately, so far, sacubitril/valsartan is not reimbursed in Poland (unlike in other European and non-European countries) for HFrEF patients, which results in underutilization of this novel treatment in Polish patients. Consequently, the experience with ARNI is limited in Poland.

Therefore, this study aims to respond to the as-yet unmet clinical need to investigate the subject of clinical experience with sacubitril/valsartan in a mid-sized HFrEF cohort from $3 \mathrm{HF}$ referral centers in central and southern Poland.

\section{Material and methods}

This observational multicenter study was conducted in 3 clinical centers in Poland (Kraków, Łódź and Warszawa) specializing in HF. The study included 89 patients suffering from chronic HFrEF. The inclusion criteria were as follows: stable (defined as at least 4 weeks without HF exacerbation) and symptomatic HF categorized as NYHA (New York Heart Association) class II-IV (ambulatory); left ventricular ejection fraction (LVEF) lower than or equal to 40\%; and optimal treatment of HFrEF according to the guidelines of the European Society of Cardiology (ESC). ${ }^{4}$ The exclusion criteria were: hypotension (systolic blood pressure (SBP) $<100$ mm Hg), renal dysfunction with estimated glomerular filtration rate (eGFR) $<30 \mathrm{~mL} / \mathrm{min} / 1.73 \mathrm{~m}^{2}$, hyperkalemia $>5.4 \mathrm{mmol} / \mathrm{L}$, history of angioedema, and thyroid dysfunction.

The comprehensive analysis of clinical, laboratory and echocardiographic parameters was performed and included the following:
- age, gender, body mass index (BMI), and systolic (SBP) and diastolic (DBP) arterial blood pressure,

- coincidence of arterial hypertension, diabetes mellitus (DM), CAD, history of myocardial infarction, atrial fibrillation (AF), renal failure, chronic obstructive pulmonary diseases (COPD), cancer, and coronary interventions such as percutaneous coronary intervention (PCI) or coronary artery bypass graft (CABG);

- electrotherapy - cardiac resynchronization therapy (CRT-D, CRT-P) and implantable cardioverter defibrillator (ICD);

- HF etiology (ischemic compared to non-ischemic) and duration of HF;

- basic laboratory results, i.e., N-terminal prohormone B-type natriuretic peptide (NT-proBNP) and high-sensitivity troponin $\mathrm{T}$, creatinine with eGFR, and potassium level;

- electrocardiography (ECG) variables: heart rhythm and heart rate (HR), QRS duration;

- selected echocardiographic results, i.e., LVEF, enddiastolic volume (EDV)/end-systolic volume (ESV) of left ventricle, right ventricular diameter (RVD), tricuspid annular plane systolic excursion (TAPSE), pulmonary artery systolic pressure (PASP), volume of left atrium;

- drugs and doses of standard HFrEF therapy: angiotensin-converting-enzyme inhibitors (ACEI), angiotensin receptor blockers (ARB), $\beta$-blockers $(\mathrm{BB})$, mineralocorticoid receptor antagonist (MRA), ivabradine, diuretics, and digoxin;

- results of six-minute walking test (6MWT);

- MAGGIC (Meta-Analysis Global Group in Chronic Heart Failure) score. ${ }^{7}$

During a one-year follow-up, the following factors were analyzed: clinically important variables (NYHA class, arterial blood pressure, HR, QRS duration, LVEF, NT-proBNP, $6 \mathrm{MWT}$, creatinine, and potassium level), and the composite endpoint (death and/or HF hospitalization).

The paper also includes an analysis of the sacubitril/ valsartan safety and tolerability profile.

\section{Initial dose of sacubitril/valsartan and increase to the target maintenance dose}

Patients enrolled in the study received a starting dose of sacubitril/valsartan as 1 tablet of 24/26 mg twice daily or 49/51 mg twice daily, as recommended. ${ }^{8}$ The sacubitril/ valsartan 24/26 mg initial dose was used in patients with $\mathrm{SBP} \geq 100-110 \mathrm{~mm} \mathrm{Hg}$, in patients not currently taking ACE-I or ARB, or taking low doses of these medicinal products, and in patients with moderate renal impairment (eGFR $30-60 \mathrm{~mL} / \mathrm{min} / 1.73 \mathrm{~m}^{2}$ ) or moderate hepatic insufficiency (Child-Pugh class B). Sacubitril/valsartan 49/51 mg was used in patients with SBP > $110 \mathrm{~mm} \mathrm{Hg}$, and normal eGFR and serum potassium level. The dose was doubled at 2-4 weeks to the target dose of 1 tablet of $97 / 103 \mathrm{mg}$ twice daily, as tolerated by the patient with 
normal pressure tolerance, normal eGFR and normal serum potassium level.

The dose was temporarily reduced or discontinued in case of symptomatic SBP $\leq 90 \mathrm{~mm} \mathrm{Hg}$, hyperkale$\mathrm{mia}>5.4 \mathrm{mmol} / \mathrm{L}$ and worsening renal function defined as eGFR $<30 \mathrm{~mL} / \mathrm{min} / 1.73 \mathrm{~m}^{2}$. Patients received sacubitril/ valsartan at least $36 \mathrm{~h}$ after discontinuing ACE inhibitor therapy.

\section{Statistical analysis}

Quantitative variables are reported as mean and standard deviation (SD) or, for non-normally distributed variables, the median and interquartile range (IQR). Normality of the variables was verified using the Shapiro-Wilk normality test. For categorical variables, the number of observations $(\mathrm{N})$ with the corresponding percentage (\%) is given.

To compare 2 independent groups, Student's t-test for quantitative variables with normal distribution or the nonparametric Mann-Whitney U test for non-normally distributed variables were used. To compare more than 2 independent groups, analysis of variance (ANOVA, for normally distributed quantitative variables) or the Kruskal-Wallis test (if the distributions of variables were different from normal) with post hoc multiple comparisons (Tukey's honest significant difference (HSD) test) was used.

For categorical variables, Pearson's $X^{2}$ test, the maximum likelihood (ML) $\chi^{2}$ test or $\chi^{2}$ test with Yates's correction was applied (regarding the expected counts in the contingency tables).

The paired sample t-test (for normally distributed quantitative variables) or the nonparametric Wilcoxon signedrank test (for non-normally distributed quantitative variables) or the McNemar-Bowker test with correction for continuity (for categorical variables) was used to compare 2 dependent groups (before and after the treatment).

Variables significant in univariate comparisons at $\mathrm{p}<0.05$ were included in the multivariable stepwise logistic regression model to determine the independent risk factors of the composite endpoint.

Taking into account the time to event (i.e., the time to death, the composite endpoint), the Kaplan-Meier survival curves were determined. To compare 2 Kaplan-Meier curves, the log-rank test was applied. The Cox proportional hazards model was used to determine the independent risk factors of the composite endpoint.

The results were considered statistically significant at $\mathrm{p}<0.05$. All the calculations were performed using the STATISTICA PL v. 13.3 package (StatSoft Inc., Tulsa, USA). The studied population was analyzed in relation to HF etiology: ischemic compared to non-ischemic and composite endpoint.

The study design was approved by the Bioethics Committee of the Jagiellonian University, Kraków, Poland (approval No. 1072.6120.55.2020).

\section{Results}

Table 1 shows the baseline characteristics of the total population and the analyzed groups of patients, divided according to HF etiology into ischemic HF ( $n=42,47 \%)$ and non-ischemic HF, caused mainly by dilated cardiomyopathy (DCM), arrhythmias or primary valvular heart diseases. Patients with non-ischemic HF were younger, had shorter duration of $\mathrm{HF}$, had more preserved renal function, larger left ventricles and lower EF, but they had higher lowdensity lipoprotein (LDL) levels. The 2 groups did not differ in terms of baseline blood pressure, HR, NYHA class, number of comorbidities, NT-proBNP levels, or baseline HF medications.

For the total population, the comparison of clinically relevant parameters between baseline and at one-year assessment is presented in Table 2. Overall, NYHA class, distance in the 6MWT, LVEF, and NT-proBNP levels significantly improved after a year of treatment. Further, we observed significant reductions in both SBP and DBP, HR, and QRS complex width. Finally, potassium levels and creatinine remained unchanged.

After a year, $20 \%(n=15)$ of patients were treated with $24 / 26 \mathrm{mg}$ of sacubitril/valsartan twice daily, $28 \%(\mathrm{n}=20)$ received $49 / 51 \mathrm{mg}$ twice daily and $52 \%(\mathrm{n}=38)$ received the maximum dose. An adverse event in the form of hypotension, hyperkalemia or worsening renal function occurred in $17.8 \%(\mathrm{n}=16), 5.6 \%(\mathrm{n}=5)$ and $4.5 \%(\mathrm{n}=4)$ of patients, respectively.

In $12 \%(\mathrm{n}=11)$ of patients, the sacubitril/valsartan dose was reduced during the study. Hypotension was the cause of the dose reduction in 10 patients (11\%) and the decrease of eGFR in 1 patient (1\%). Sacubitril/valsartan was discontinued in $10 \%(n=9)$ and hypotension was the main cause $(4.5 \%, \mathrm{n}=4)$, in 1 patient at dose $49 / 51 \mathrm{mg}$ twice daily and in 3 patients at 97/103 mg twice daily. One patient (1\%) discontinued treatment due to worsening renal function (drug dose 49/51 mg twice daily) and 4 patients (4.5\%) due to hyperkalemia - 1 patient at a dose of $49 / 51 \mathrm{mg}$ twice daily and 3 patients at a dose of 97/103 mg twice daily.

The population that received the highest dose of sacubitril/valsartan compared to patients receiving the lowest or intermediate dose had the shortest history of $\mathrm{HF}$ (respectively 70 months vs 157 months vs 81 months; $\mathrm{p}=0.001)$, the lowest number of visits to primary care ( 4 vs 6 vs $4.5 ; \mathrm{p}=0.005$ ), the highest SBP (respectively $123 \mathrm{~mm} \mathrm{Hg}$ vs $108 \mathrm{~mm} \mathrm{Hg}$ vs $113 \mathrm{~mm} \mathrm{Hg}$; = 0.002) and DBP (75 mm Hg vs $66 \mathrm{~mm} \mathrm{Hg}$ vs $72 \mathrm{~mm} \mathrm{Hg}$; = 0.002), and the lowest initial NT-proBNP (respectively $2620 \mathrm{pg} / \mathrm{mL}$ vs $4446 \mathrm{pg} / \mathrm{mL}$ vs $4417 \mathrm{pg} / \mathrm{mL}$; $\mathrm{p}=0.003)$.

During a one-year follow-up, 8 patients died (8.9\%); the mean time to death was $5.88 \pm 4.16$ months. One of non-survivors discontinued the treatment of sacubitril/valsartan 1 month before death. The MAGGIC score in the whole group was $24 \pm 5.4$. This implies one-year probability of death of approx. $14.7 \%$, which is much higher 
Table 1. Baseline characteristics and related to etiology. Data is presented as mean \pm standard deviation (SD), median (interquartile range - IQR) or number (percentage) in variables with non-parametric distribution

\begin{tabular}{|c|c|c|c|c|}
\hline Parameter & Total population, $n=89$ & Non-ischemic HF, $n=47$ & Ischemic HF, $\mathrm{n}=42$ & $\mathrm{p}$-value \\
\hline Age [years] & $62(56-68)$ & $59(43-66)$ & $63(59-72)$ & 0.002 \\
\hline Male sex, n (\%) & $73(82)$ & $34(72.3)$ & $39(92.9)$ & 0.025 \\
\hline BMI $\left[\mathrm{kg} / \mathrm{m}^{2}\right]$ & $27.34 \pm 4 / 6$ & $26.95 \pm 4.26$ & $27.79 \pm 4.91$ & 0.39 \\
\hline HF duration [months] & $72(32-133)$ & $50.5(12-120)$ & $85.5(42-143)$ & 0.03 \\
\hline $\mathrm{SBP}[\mathrm{mm} \mathrm{Hg}]$ & $116.9 \pm 14.1$ & $114.94 \pm 14.51$ & $119.1 \pm 13.5$ & 0.17 \\
\hline $\mathrm{DBP}[\mathrm{mm} \mathrm{Hg}]$ & $72.5 \pm 8.5$ & $72.30 \pm 8.19$ & $72.76 \pm 8.94$ & 0.85 \\
\hline NYHA class & $3(3-3)$ & $3(3-3)$ & $3(3-3)$ & 0.98 \\
\hline Hospitalization HF, n (\%) & $48(54)$ & $23(48.9)$ & $25(59.5)$ & 0.32 \\
\hline Atrial fibrillation, $\mathrm{n}(\%)$ & $36(40)$ & $18(38.3)$ & $18(42.9)$ & 0.66 \\
\hline Diabetes, n (\%) & $36(40)$ & $19(40.4)$ & $17(40.5)$ & 0.99 \\
\hline Hypertension, n (\%) & $52(58)$ & $23(48.9)$ & $29(69)$ & 0.055 \\
\hline Dyslipidemia, n (\%) & $49(55)$ & $22(46.8)$ & $27(64.3)$ & 0.09 \\
\hline COPD, n (\%) & $8(9)$ & $2(4.3)$ & $6(14.3)$ & 0.2 \\
\hline NT-proBNP [pg/mL] & $2600(914-4783)$ & $2858(1017-4967)$ & $2186(797-4783)$ & 0.73 \\
\hline Hemoglobin [g/dL] & $13.8 \pm 1.88$ & $13.97 \pm 2.13$ & $13.6 \pm 1.6$ & 0.4 \\
\hline $\mathrm{Na}[\mathrm{mmol} / \mathrm{L}]$ & $139.9 \pm 2.78$ & $140.1 \pm 2.65$ & $139.7 \pm 2.94$ & 0.5 \\
\hline $\mathrm{K}[\mathrm{mmol} / \mathrm{L}]$ & $4.49 \pm 0.39$ & $4.46 \pm 0.37$ & $4.52 \pm 0.41$ & 0.53 \\
\hline Creatinine $[\mu \mathrm{mol} / \mathrm{L}]$ & $94.6(81-113)$ & $91(77-108)$ & $105.1(85-129)$ & 0.03 \\
\hline $\mathrm{eGFR}\left[\mathrm{mL} / \mathrm{min} / 1.73 \mathrm{~m}^{2}\right]$ & $70.9 \pm 22.7$ & $76.22 \pm 22.36$ & $65.1 \pm 21.9$ & 0.02 \\
\hline BUN $[\mathrm{mmol} / \mathrm{L}]$ & $12.3(8-17.5)$ & $13.2(7.95-19.8)$ & $13.4(6.7-26.0)$ & 0.69 \\
\hline Glucose $[\mathrm{mmol} / \mathrm{L}]$ & $5.7(5.1-6.8)$ & $5.8(5-7.1)$ & $5.5(5.1-6.6)$ & 0.45 \\
\hline LDL [mmol/L] & $2.23 \pm 0.88$ & $2.43 \pm 0.97$ & $2.02 \pm 0.72$ & 0.04 \\
\hline Bilirubin [mg/dL] & $20.7(14.3-39.4)$ & $17.3(13-37.9)$ & $26.7(17-47.6)$ & 0.17 \\
\hline $\operatorname{LVD}[\mathrm{mm}]$ & $66(60-72)$ & $68.66 \pm 9.57$ & $63.7 \pm 9.1$ & 0.014 \\
\hline EDV LV [mL] & $221.2 \pm 90.3$ & $221.9 \pm 106.1$ & $220.1 \pm 65.1$ & 0.69 \\
\hline ESV LV [mL] & $171.2 \pm 81.4$ & $175.55 \pm 96.24$ & $165 \pm 55.1$ & 0.66 \\
\hline RVD prox. [mm] & $34.9 \pm 6.02$ & $34.87 \pm 6.5$ & $35.1 \pm 5.6$ & 0.88 \\
\hline LA vol. [mL] & $133.6 \pm 51.4$ & $129.25 \pm 45.28$ & $136.7 \pm 56.2$ & 0.8 \\
\hline LVEF [\%] & $23.6 \pm 6.7$ & $22.25 \pm 6.85$ & $25.05 \pm 6.24$ & 0.02 \\
\hline TAPSE $[\mathrm{mm}]$ & $16.7 \pm 4.2$ & $16.5 \pm 3.96$ & $16.9 \pm 4.5$ & 0.6 \\
\hline PASP [mm Hg] & $43.3 \pm 13.19$ & $42.5 \pm 13.9$ & $44.1 \pm 12.6$ & 0.69 \\
\hline 6MWT distance $[\mathrm{m}]$ & $353.2 \pm 99.6$ & $357.64 \pm 96.23$ & $347.9 \pm 105.6$ & 0.73 \\
\hline HR [bpm] & $74.4 \pm 7.4$ & $77.7 \pm 12.9$ & $75.9 \pm 8.5$ & 0.86 \\
\hline QRS duration [ms] & $114(102-140)$ & $110(100-140)$ & $120(108-128.5)$ & 0.83 \\
\hline Prior ACEI/ARB, n (\%) & $87(98)$ & $45(95.7)$ & $42(100)$ & 0.98 \\
\hline$\beta$-blockers, n (\%) & $87(98)$ & $46(97.9)$ & $41(97.6)$ & 0.98 \\
\hline MRA, n (\%) & $80(90)$ & $43(91.5)$ & $37(88.1)$ & 0.86 \\
\hline Ivabradine, n (\%) & $16(18)$ & $7(15.2)$ & $9(21.4)$ & 0.45 \\
\hline Loop diuretic, n (\%) & $73(85)$ & $37(87.2)$ & $36(87.8)$ & 0.67 \\
\hline Digoxin, n (\%) & $16(18)$ & $10(21.3)$ & $6(14.3)$ & 0.39 \\
\hline MAGGIC score & $24 \pm 5.4$ & $23.1 \pm 4.7$ & $25 \pm 6.04$ & 0.1 \\
\hline ICD at baseline, $\mathrm{n}(\%)$ & $36(40)$ & $14(40.4)$ & $22(52.4)$ & 0.26 \\
\hline CRT at baseline, $n$ (\%) & $15(17)$ & $6(12.8)$ & $9(21.4)$ & 0.28 \\
\hline
\end{tabular}

ACEI - angiotensin-converting-enzyme inhibitors; ARB - angiotensin receptor blockers; BMI - body mass index; BUN - blood urea nitrogen; COPD - chronic obstructive pulmonary disease; CRT - cardiac resynchronization therapy; DBP - diastolic blood pressure; DM - diabetes mellitus; eGFR - estimated glomerular filtration rate; HF - heart failure; HR - heart rate; ICD - implantable cardioverter defibrillator; LDL - low-density lipoprotein; LVD - left ventricle diameter; LVEF - left ventricle ejection fraction; MRA - mineralocorticoid antagonist; NT-proBNP - N-terminal prohormone B-type natriuretic peptide; SBP - systolic blood pressure; EDV LV - end diastolic volume - left ventricle; ESV LV - end systolic volume - left ventricle; RVD - reference vessel diameter; LA vol. - left atrial volume; TAPSE - tricuspid annular plane systolic excursion; PASP - pulmonary artery systolic pressure; 6MWT - six-minute walk test; MAGGIC - Meta-Analysis Global Group in Chronic Heart Failure. 
Table 2. Comparison of clinically important variables after 1 year of treatment with sacubitril/valsartan. Data is presented as mean \pm standard deviation (SD), median (interquartile range - IQR) or number (percentage) in variables with non-parametric distribution

\begin{tabular}{|l|c|c|c|}
\hline \multicolumn{1}{|c|}{ Parameter } & Baseline $(\mathrm{n}=73)$ & After 1 year of treatment $(\mathrm{n}=73)$ & $\mathrm{p}$-value \\
\hline NYHA class & $3(3-3)$ & $2(1-2)$ & $<0.001$ \\
\hline SBP $[\mathrm{mm} \mathrm{Hg}]$ & $117.2 \pm 13.9$ & $109.9 \pm 13.4$ & $<0.001$ \\
\hline DBP $[\mathrm{mm} \mathrm{Hg]}$ & $72.7 \pm 8.3$ & $68.3 \pm 9.9$ & $<0.001$ \\
\hline HR $[\mathrm{bpm}]$ & $76(70-80)$ & $75(68-79)$ & $<0.005$ \\
\hline QRS duration $[\mathrm{ms}]$ & $115(102-140)$ & $110(107-135)$ & $<0.01$ \\
\hline LVEF $[\%]$ & $23.8 \pm 6.5$ & $27 \pm 6.4$ & $<0.001$ \\
\hline K $[\mathrm{mmmol} / \mathrm{L}]$ & $4.4 \pm 0.4$ & $4.5 \pm 0.38$ & 0.88 \\
\hline Creatinine $[\mathrm{\mu mol} / \mathrm{L}]$ & $95(81-113)$ & $101(86-115)$ & 0.21 \\
\hline NT-proBNP $[\mathrm{pg} / \mathrm{mL}]$ & $2600(969.2-4542)$ & $1628(679-3009)$ & $<0.001$ \\
\hline 6MWT distance $[\mathrm{m}]$ & $337.3 \pm 96.9$ & $423.8 \pm 71.9$ & $<0.001$ \\
\hline
\end{tabular}

DBP - diastolic blood pressure; HR - heart rate; LVEF - left ventricle ejection fraction; NT-proBNP - N-terminal prohormone B-type natriuretic peptide; SBP - systolic blood pressure; NYHA - New York Heart Association; 6MWT - six-minute walk test.

than the observed one-year mortality in the studied population. Furthermore, the MAGGIC score was similar in non-survivors and survivors $(25.7 \pm 5.7$ vs $23.9 \pm 5.3$; $\mathrm{p}=0.37)$. The Kaplan-Meier curves for death in the total population and related to HF etiology $(\mathrm{p}>0.05)$ is presented in Fig. 1 and Fig. 2.

During a one-year follow-up, in 24 patients (26.9\%), the composite endpoint was reported. These patients had a higher level of NT-proBNP (4488.5 [2599-7129.5] pg/mL vs 2049.5 [746-3889.5] pg/mL, $\mathrm{p}=0.002)$, more HF hospitalizations in the last 12 months (median 2 [1-3] vs 0 [0-1]; $\mathrm{p}=0.0001$ ) and lower SBP $(111.3 \pm 12.5 \mathrm{~mm} \mathrm{Hg}$ vs $118.5 \pm 13.9 \mathrm{~mm} \mathrm{Hg}$; $\mathrm{p}=0.028)$, distance in $6 \mathrm{MWT}(301.6 \pm 101.3 \mathrm{~m}$ vs
$367.1 \pm 95.3 \mathrm{~m} ; \mathrm{p}=0.046)$ and TAPSE $(16.6 \pm 2.65 \mathrm{~mm}$ vs $17.5 \pm 4.46 \mathrm{~mm} ; \mathrm{p}=0.004)$. These parameters indicated more advanced HF in these patients. In analysis regarding HF etiology, the composite endpoint occurred in 11 patients with ischemic etiology and in 13 with non-ischemic $(\mathrm{p}=0.83)$. The time to composite endpoint was $3.32 \pm 2.03$ months for ischemic etiology of HF and $4.44 \pm 3.95$ months for nonischemic etiology. The multivariable analysis revealed 3 independent variables for risk of composite endpoint (Table 3): history of HF hospitalization, TAPSE and ACEI treatment. According to ANOVA analysis, only RVD in ischemic etiology of HF was significant for risk of composite endpoint $(\mathrm{p}=0.0452)$ (Fig. 3).

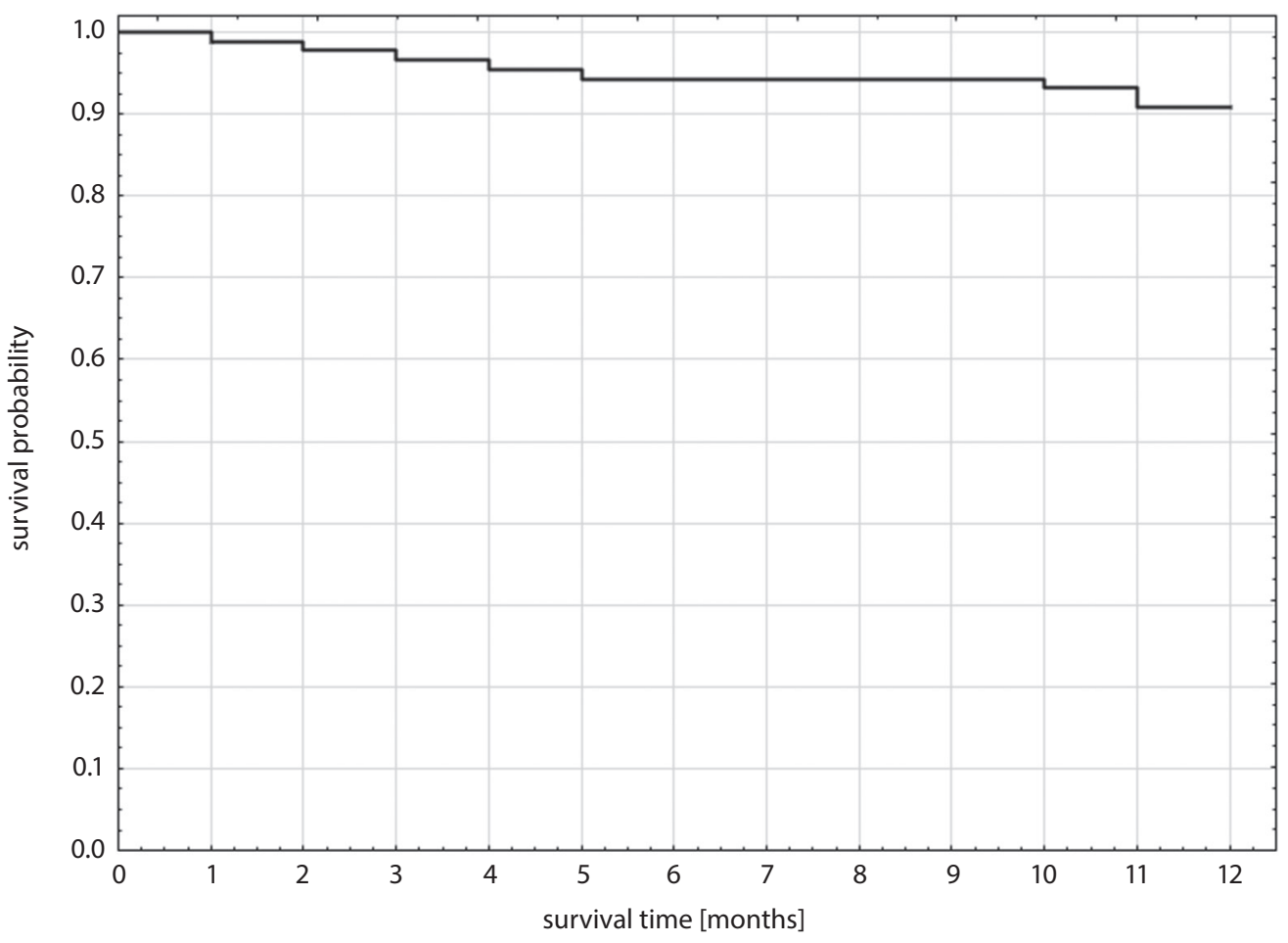

Fig. 1. Kaplan-Meier curve of death during 1 year in the studied population 

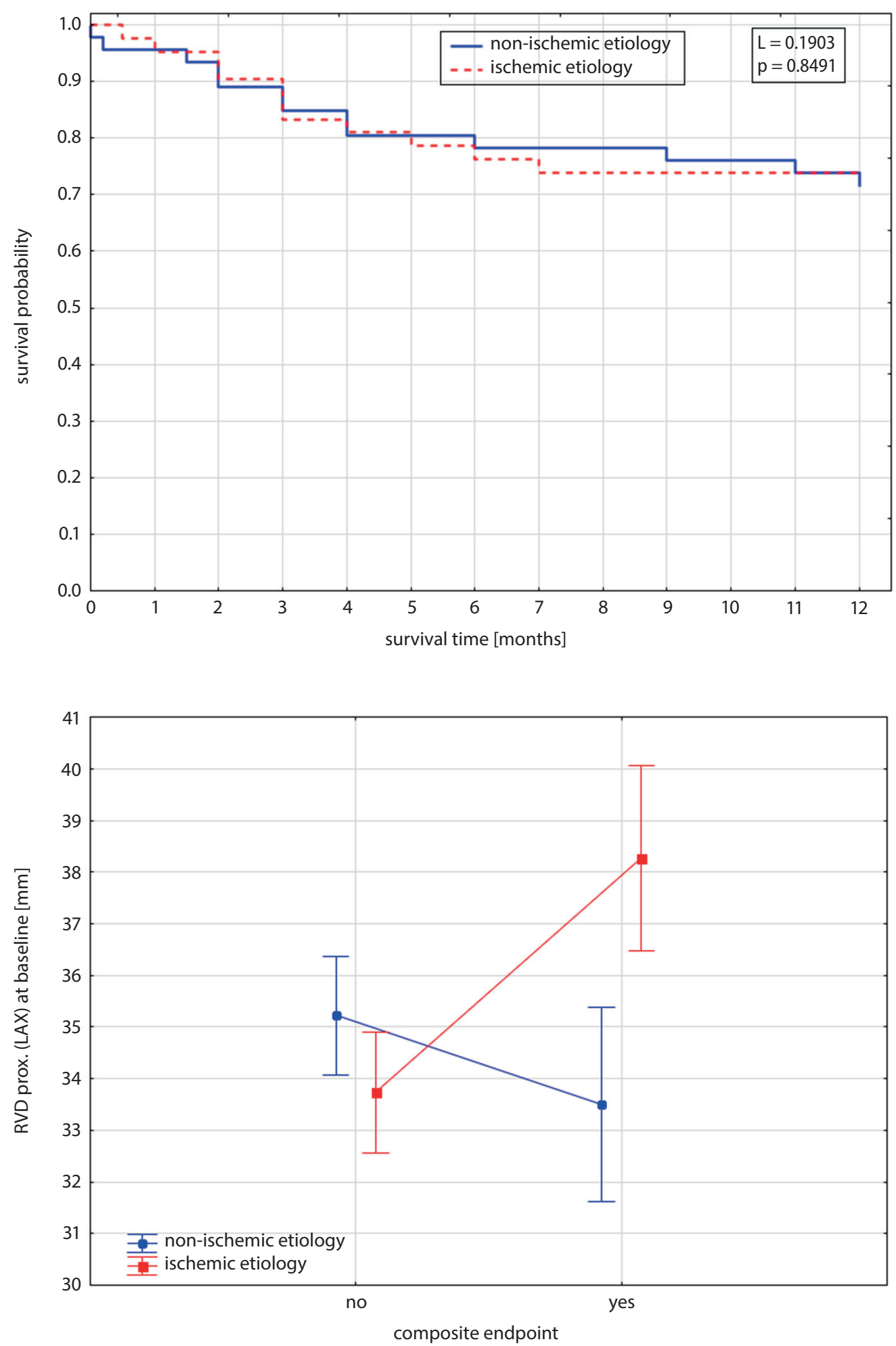

Fig. 2. Kaplan-Meier curve of composite endpoint (death and/or HF hospitalization) during 1 year in the studied population related to etiology of HF
Fig. 3. Two-way ANOVA results for RVD prox. (LAX) at baseline - interaction effect between etiology and composite endpoint (marginal means \pm standard error $(\mathrm{SE}) ; \mathrm{p}=0.0453$ )

\section{Discussion}

We present the first real-life observation of one-year treatment with sacubitril/valsartan in a Polish HFrEF population. The studied population represents a typical HFrEF cohort. Of note, we report a relatively high proportion of DCM patients (52.5\% vs $40 \%$ in PARADIGM-HF), lower EF (our population $-23.5 \pm 8.9$ vs PARADIGM-HF
- $29.6 \pm 6.1 \%$ and higher levels of NT-proBNP (ours - $2600 \mathrm{pg} / \mathrm{mL}$ vs $1631 \mathrm{pg} / \mathrm{mL}$ ); such results reflect more advanced HFrEF and probably the fact that our centers serve as regional referral centers for more sophisticated diagnostic work-up (e.g., referrals for heart transplant or mechanical circulatory support in DCM or hemodynamically compromised patients). ${ }^{6}$ More advanced HFrEF in our study as well as in the first report on Polish patients 
Table 3. Results of multivariable analysis for the composite endpoint

\begin{tabular}{|l|c|c|c|c|}
\multicolumn{1}{|c|}{ Variable } & p-value & OR & \multicolumn{2}{|c|}{$95 \%$ CI for OR } \\
\hline History of HF hospitalization & 0.001 & 2.194 & 1.397 & 3.448 \\
\hline TAPSE at baseline & 0.035 & 0.826 & 0.691 & 0.987 \\
\hline ACEI before ARNI & 0.011 & 0.176 & 0.046 & 0.672 \\
\hline Intercept & 0.144 & $x$ & $x$ & $x$ \\
\hline
\end{tabular}

OR - odds ratio; 95\% Cl - 95\% confidence interval; HF - heart failure; TAPSE - tricuspid annular plane systolic excursion; ACEI - angiotensin-convertingenzyme inhibitor; ARNI - angiotensin receptor neprilysin inhibitors.

from $2018^{9}$ in comparison to the PARADIGM-HF baseline characteristics probably results from the fact that tertiary cardiac centers, with the greatest HF expertise, decided to start ARNI treatment in Poland.

Overall, we observed very favorable outcomes after 1 year of treatment with sacubitril/valsartan, which was consistent in various parameters of clinical interest, such as improvements in NYHA class, EF and distance in 6MWT, as well as substantial reduction of NT-proBNP levels. Reassuringly, and similarly as in other ARNI trials, including PARADIGM-HF, we did not observe safety issues with ARNI, i.e., the substantial reduction of both SBP and DBP was asymptomatic in the great majority of patients, and the much feared worsening of renal function did not occur (stable levels of creatinine and potassium).

Although the study population was relatively small compared to large multi-center randomized controlled trials or ESC-initiated registries, we report here a real-life and probably the largest population of HFrEF patients treated with ARNI in Poland. For the first time we report a one-year follow-up, which reassuringly is similar to other studied populations in real-world studies. ${ }^{10-14}$ This and other studies showed a clear benefit of sacubitril/valsartan treatment of HFrEF patients, which directly translates into improvement in clinical, echocardiographic and laboratory indices. We sincerely hope that the increasing number of papers showing effectiveness of sacubitril/valsartan will pave the way for widespread utilization of this treatment in Polish patients, which currently is limited due to non-medical reasons (unbearable financial cost for the majority of patients).

Sacubitril/valsartan is recommended in the ESC guidelines for further reduction in the risk of hospitalization or death in patients with HFrEF if symptoms continue despite optimal treatment with ACEI/ARB, $\beta$-blockers and mineralocorticoid antagonists. At baseline, the studied population was optimally treated in terms of the class of the standard therapy of ACEI/ARB, BB and MRA (Table 1). After 1 year, only ivabradine was more frequently used (from $18 \%$ at baseline to $29 \%, \mathrm{p}=0.077$ ). However, the target doses of $50 \%$ or more were obtained for BB from $66 \%$ at baseline to $63 \%$ after a year, for MRA it was stable at $77 \%$, and for ivabradine it varied from $54 \%$ at baseline to $69 \%$. It should be emphasized that in comparison to the observational QUALIFY registry for ambulatory Polish patients suffering from chronic $\mathrm{HF},{ }^{15}$ both prescription of standard
HFrEF therapy and ivabradine and the proportion of target doses are higher in our study, but still suboptimal.

For ARNI, the TITRATION study indicated that $75.9 \%$ of randomized patients achieved 'treatment success', defined as achieving and maintaining a dose of sacubitril/ valsartan of 97/103 mg twice daily without any dose interruption/down-titration over 12 weeks. ${ }^{16}$ In our study, after a year, $80 \%$ of patients were on $50 \%$ or more of the target dose of sacubitril/valsartan (49/51 mg or 97/103 mg twice daily). In the PROVE-HF study, after a year of treatment, $65 \%$ of patients received the maximum dose of sacubitril/ valsartan, $21 \%$ received a moderate dose and $14 \%$ received the lowest dose. ${ }^{17}$ In our study, the involvement of individual doses represents $52 \%, 28 \%$ and $20 \%$, respectively, and was comparable in the moderate dose (49/51 mg) to the PROVE-HF study. ${ }^{17}$ More frequent use of lower doses in our population in relation to the PROVE-HF populations may have resulted from lower SBP and DBP $(116 \pm 14 \mathrm{~mm} \mathrm{Hg}$ and $72 \pm 8 \mathrm{~mm} \mathrm{Hg}$ vs $124.5 \pm 16 \mathrm{~mm} \mathrm{Hg}$ and $76 \pm 10.3 \mathrm{~mm} \mathrm{Hg}$, respectively) and more advanced HF (NT-proBNP 2600 pg/mL vs 816 pg/mL, respectively). ${ }^{17}$

In our study, patients treated with the maximum target dose constituted the least burdened population at baseline (eGFR $75.91 \mathrm{~mL} / \mathrm{min} / 1.73 \mathrm{~m}^{2}$, SBP $123 \mathrm{~mm} \mathrm{Hg}$ ). It allowed us to use the maximum doses, which further enhanced the therapeutic effects in comparison to the groups at lower doses. Thus, the earlier incorporation of the drug, as well as treatment with higher doses, has a beneficial effect on the prognosis of patients with HFrEF.

Hypotension, hyperkalemia and worsening renal function were the most frequent adverse events in our and the PROVE-HF study. Hypotension was observed with a comparable frequency in the studied population and in the PROVE-HF study (17.8\% compared to $17.6 \%$, respectively). ${ }^{17}$ It should be emphasized that hyperkalemia and worsening renal function were less frequently observed in our study than in the PROVE-HF study (5.6\% and $4.5 \%$ compared to $13.2 \%$ and $12.3 \%) .{ }^{17}$ Moreover, no statistically significant differences were observed in potassium and creatinine values in our population at the beginning of the study and after 1 year. However, the most common reasons for discontinuation of therapy were hypotension, hyperkalemia and worsening kidney function in both our study and PARADIGM-HF, and at a similar level (11\% compared to $11.4 \%$, respectively). ${ }^{6}$ 
At baseline, a little more than half of the population had devices (ICD or CRT in 57\%), and in the one-year followup, 14 patients subsequently received an ICD (8) or CRT (6). That is even more than in the QUALIFY population (28.7\%); however, there is still a large need for invasive procedures in the HFrEF population in our country.

In the studied population, AF was highly prevalent (36/89 patients), including 18 (38.3\%) out of 47 non-ischemic HF and 18 (42.9\%) out of 42 ischemic HF patients. In the AF population, 22 (61\%) had severe reduction in LVEF $\leq 25 \%$ and 16 (44\%) were older than 65. Regardless of HF etiology, there were no differences of the drug therapy, including $\beta$-blockers. Digoxin was used in 16 patients (18\%). During the one-year observation, no ablation procedure was performed.

The mean HR in the AF group was $81 \mathrm{bpm}$, while in the sinus rhythm group it was $75 \mathrm{bpm}$. At baseline, $35 \mathrm{pa}-$ tients with AF (97\%) were taking $\beta$-blockers and after 1 year - all patients; however, 13 (36\%) were treated less than 50\% of the target $\beta$-blockers. After 1 year of sacubitril/valsartan treatment, a change in $\beta$-blockers treatment was observed in 5 patients. Three patients had reduced their $\beta$-blockers dose (bradyarrhythmia), 1 discontinued the treatment due to peripheral artery disease (PAD), and 1 patient had increased the dose above $50 \%$ of the target dose. During the one-year follow-up, in 13 AF patients (36\%), the composite endpoint was reported, including 3 deaths (8\%).

In the studied population, patients who died (9.09\%) had a higher level of NT-proBNP (3337 [3243-5631] pg/mL vs 1337.5 [653.5-2231.5] pg/mL; $\mathrm{p}=0.002)$ and blood urea nitrogen - BUN (27 [21-34] mmol/L vs 10.2 [6.7-21] mmol/L; $\mathrm{p}=0.0056)$, lower distance in the 6MWT $(273.2 \pm 105.1 \mathrm{~m}$ vs $431.3 \pm 85.5 \mathrm{~m} ; \mathrm{p}=0.039)$, and a larger RVD in echocardiography measured in the parasternal longitudinal axis $(40.86 \pm 4.71 \mathrm{~mm}$ vs $34.3 \pm 5.85 \mathrm{~mm}, \mathrm{p}=0.0055)$. These parameters indicated the more advanced HF in the nonsurvival population. The integer risk score of approx. 24 locates our population between the $3^{\text {rd }}$ and $4^{\text {th }}$ risk group with estimated one-year probability of death between $13.4 \%$ and $16 \%{ }^{7}$ Thus, the MAGGIC score clearly overestimated the one-year probability of death in our population (true one-year mortality in our population $-9.09 \%$ compared to estimated one-year mortality $-14.7 \%){ }^{7}$ Apart from numerous factors that may be responsible for this inaccuracy, such as the relatively small population size (which may distort statistical calculations), the majority of patients with non-ischemic HF (with usually worse prognosis in ischemic HF), younger age of our population than the original MAGGIC cohort, etc., the favorable effect of sacubitril/ valsartan should also be taken into account.

The composite endpoint (cardiovascular mortality and hospitalization) after a year occurred in $17.3 \%$ in the ESCEORP-HFA Heart Failure Long-Term Registry, ${ }^{18}$ and in our study in $27.3 \%$. However, it should be pointed out that the components of the composite endpoint in our study are different (death and HF hospitalization). It should also be noted that there were differences in hospitalization due to decompensation, in $13 \%$ of patients in the abovementioned Registry compared to $27.3 \%$ in the present population. It seems that the increased number of hospitalizations in our study is a result of more advanced HF in the studied population and the inadequately organized outpatient care in our country (the routine treatment of patients with decompensation of HF in hospital). Mortality in our population was $9.09 \%$ and was higher than in the Registry after a year of treatment of sacubitril/valsartan, which was $8.8 \%{ }^{18}$ Ischemic etiology $(5.6 \%, \mathrm{n}=5)$ was a more frequent cause of death in our population than non-ischemic etiology $(3.3 \%$, $\mathrm{n}=3$ ), which is consistent with other studies that indicate a worse prognosis for patients with ischemic HF etiology.

From multivariable analysis independent variables for risk of composite endpoint were revealed (history of HF hospitalization, TAPSE and ACEI treatment at baseline), which indicate more advanced $\mathrm{HF}$ and worse prognosis in ACEI-naive patients, and according to ANOVA analysis, in ischemic HF only the RVD was important for risk of composite endpoint. It is well known that the right ventricle dilates in end-stage disease, and is a predictor of poor outcome in HF. ${ }^{19,20}$ However, recently Correale et al. observed in a real-life population with chronic HFrEF improvement of right ventricular function under sacubitril/valsartan treatment. ${ }^{21}$

\section{Limitations}

There are several potential limitations to the present study that should be acknowledged. Firstly, the size of the study population is small; however, ARNI treatment in Poland in not reimbursed, which results in unbearable cost of treatment for the majority of patients in need. Secondly, the fact that patients were recruited in the referral centers may slightly distort the typical HFrEF patients in Poland, which in fact may be less severe. Thirdly, the observation period of 12 months seems to be relatively short; nevertheless, the composite endpoint occurred in more than a quarter of patients. Fourthly, the mean age of our study population is at least a decade younger than a typical (real-world) HF cohort. Fifthly, a great majority of our patients have long-standing HF. Consequently, new-onset (or de novo) HF patients are under-represented. Also, this was an observational study without a control group. The lack of direct His bundle pacing in our patients with chronic AF, which is more and more widely used in this group of patients, ${ }^{22}$ may also be considered as a limitation of our work.

\section{Conclusions}

In summary, we present the first one-year observation of real-life HFrEF Polish patients treated with sacubitril/ valsartan with clinical improvement and good tolerability. 
We confirmed that non-survivors had more advanced HF, so the initiation and uptitration of sacubitril/valsartan should be performed early in HFrEF.

\section{ORCID iDs}

Małgorzata Lelonek (1) https://orcid.org/0000-0003-0756-5541 Sylwia Wiśniowska-Śmiałek (10) https://orcid.org/0000-0002-7563-6586 Paweł Rubiś (10) https://orcid.org/0000-0002-6979-3411 Izabela Nowakowska (1) https://orcid.org/0000-0002-3729-3359 Agnieszka Pawlak (i) https://orcid.org/0000-0001-9032-9130

\section{References}

1. Redfield MM, Jacobsen SJ, Burnett JC, Mahoney DW, Bailey KR, Rodeheffer RJ. Burden of systolic and diastolic ventricular dysfunction in the community: Appreciating the scope of the heart failure epidemic. JAMA. 2003;289(2):194-202.

2. Bleumink GS, Knetsch AM, Sturkenboom MCJM, et al. Quantifying the heart failure epidemic - prevalence, incidence rate, lifetime risk and prognosis of heart failure: The Rotterdam Study. Eur Heart J. 2004;25(18):1614-1619.

3. Seferovic PM, Jankowska E, Coats AJS, et al; Task Force of the HFA Atlas, and the ESC Atlas of Cardiology leadership, developed in collaboration with the National Heart Failure Societies of the ESC member and ESC affiliated member countries. The Heart Failure Association Atlas: Rationale, objectives and methods. Eur J Heart Fail. 2020;22(4):638-645. doi:10.1002/ejhf.1768

4. Ponikowski P, Voors AA, Anker SD, et al; ESC Scientific Document Group. 2016 ESC Guidelines for the diagnosis and treatment of acute and chronic heart failure: The Task Force for the diagnosis and treatment of acute and chronic heart failure of the European Society of Cardiology (ESC). Developed with the special contribution of the Heart Failure Association (HFA) of the ESC. Eur Heart J. 2016;37(27):2129-2200.

5. Volterrani M, lellamo F, Senni M, Piepoli MF. Therapeutic options of angiotensin receptor neprilysin inhibitors (ARNis) in chronic heart failure with reduced ejection fraction: Beyond RAAS and sympathetic nervous system inhibition. Int J Cardiol. 2017;226:132-135.

6. McMurray JJ, Packer M, Desai AS, et al; PARADIGM-HF Investigators and Committees. Angiotensin-neprilysin inhibition versus enalapril in heart failure. N Engl J Med. 2014;371(11):993-1004.

7. Pocock SJ, Ariti CA, McMurray JJ, et al; Meta-Analysis Global Group in Chronic Heart Failure. Predicting survival in heart failure: A risk score based on 39372 patients from 30 studies. Eur Heart J. 2013; 34(19):1404-1413.

8. Entresto ${ }^{\mathbb{B}}$ Summary of Product Characteristics. https://www.novartis. pl/system/files/product-info/entresto_chpl_2020-09.pdf. Accessed on September 1, 2020.

9. Kałużna-Oleksy M, Kolasa J, Migaj J, et al. Initial clinical experience with the first drug (sacubitril/valsartan) in a new class: Angiotensin receptor neprilysin inhibitors in patients with heart failure with reduced left ventricular ejection fraction in Poland. Kardiol Pol. 2018; 76(2):381-387.
10. Greene SJ, Butler J, Albert NM, et al. Medical therapy for heart failure with reduced ejection fraction: The CHAMP Registry. J Am Coll Cardiol. 2018;72(4):351-366.

11. Martens P, Lambeets S, Lau CW, Dupont M, Mullens W. Impact of sacubitril/valsartan on heart failure admissions: Insights from realworld patient prescriptions. Acta Cardiol. 2019;74(2):115-122. doi:10. 1080/00015385.2018.1473825

12. Wachter R, Viriato D, Klebs E, et al. Early insights into the characteristics and evolution of clinical parameters in a cohort of patients prescribed sacubitril/valsartan in Germany. Postgrad Med. 2018;130(3): 308-316.

13. Moliner-Abos C, Rivas-Lasarte M, Besora JP, et al. Sacubitril/valsartan in real-life practice: Experience in patients with advanced heart failure and systematic review. Cardiovasc Drug Ther. 2019:33(3):307-314.

14. Bastien N, Haddad H, Bergeron S, et al. The PARASAIL study: Patient reported outcomes from the Canadian real-world experience use of sacubitril/valsartan in patients with heart failure and reduced ejection fraction. Can J Cardiol. 2017;33:S162-S163.

15. Opolski G, Ozierański K, Lelonek M, Balsam P, Wilkins A, Ponikowski P; On Behalf Of The Polish Qualify Investigators. Adherence to systolic heart failure guidelines in ambulatory care in Poland: Data from the international QUALIFY survey. Pol Arch Med Wewn. 2017;127(10): 657-665. doi:10.20452/pamw.4083

16. Senni M, McMurray JJV, Wachter R, at al. Initiating sacubitril/valsar$\tan$ (LCZ696) in heart failure: Results of TITRATION, a double-blind, randomized comparison of two uptitration regimens. Eur J Heart Fail. 2016;18(9):1193-1202. doi:10.1002/ejhf.548

17. Januzzi JL, Prescott MF, Butler J, et al; PROVE-HF Investigators. Association of change in $\mathrm{N}$-terminal pro-B-type natriuretic peptide following initiation of sacubitril-valsartan treatment with cardiac structure and function in patients with heart failure with reduced ejection fraction. JAMA. 2019;322(11):1085-1095. doi:10.1001/jama.2019.12821

18. Kapelios CJ, Lainscak M, Savarese G, et al; Heart Failure Long-Term Registry Investigators. Sacubitril/valsartan eligibility and outcomes in the ESC-EORP-HFA Heart Failure Long-Term Registry: Bridging between European Medicines Agency/Food and Drug Administration label, the PARADIGM-HF trial, ESC guidelines, and real world. Eur J Heart Fail. 2019;21(11):1383-1397.

19. Raina A, Meeran T. Right ventricular dysfunction in left-sided heart failure with preserved versus reduced ejection fraction. Curr Heart Fail Rep. 2018;15:94-105.

20. Dini FL, Carluccio E, Simioniuc A, et al; Network Labs Ultrasound (NEBULA) in Heart Failure Study Group. Right ventricular recovery during follow-up is associated with improved survival in patients with chronic heart failure with reduced ejection fraction. Eur $J$ Heart Fail. 2016;18(12):1462-1471.

21. Correale M, Adriana Mallardi A, Pietro Mazzeo P, et al. Sacubitril/ valsartan improves right ventricular function in a real-life population of patients with chronic heart failure: The Daunia Heart Failure Registry. Int J Cardiol Heart Vasc. 2020;2(27):100486. doi:10.1016/j. ijcha.2020.100486

22. Sławuta A, Mazur G, Małecka B, Gajek J. Permanent His bundle pacing: An optimal treatment method in heart failure patients with $\mathrm{AF}$ and narrow QRS. Int J Cardiol. 2016;214:451-452. doi:10.1016/j.ijcard. 2016.04.022 